\title{
EL CONTROL DE ARVENSES EN LA PRODUCTIVIDAD Y CALIDAD DEL PASTO LLANERO ${ }^{1}$
}

\author{
Valentín Alberto Esqueda-Esquivel ${ }^{2}$, Maribel Montero-Lagunes ${ }^{3}$, Francisco Indalecio Juárez-Lagunes ${ }^{4}$
}

\begin{abstract}
RESUMEN
El control de arvenses en la productividad y calidad del pasto Llanero. De agosto de 2003 a diciembre de 2004 se condujo un experimento durante dos ciclos de lluvias en el municipio de Cotaxtla, Veracruz, México, con el objetivo de determinar el efecto del control de malezas en la producción y calidad del pasto Llanero. Se utilizó el diseño experimental de bloques al azar con cuatro repeticiones. Los tratamientos evaluados fueron: 1. Picloram (ácido 4-amino-3,5,6-tricloro2-piridincarboxílico) + fluroxipir (ácido 4-amino-3,5-dicloro-6-fluoro-2-piridiloxiacético) (40 g + $40 \mathrm{~g} / 1001$ agua), 2. Picloram + 2,4-D (ácido 2,4-diclorofenoxiacético) (64 g + $240 \mathrm{~g} / 1001$ agua) 3. Chapeo con machete y 4. Testigo sin aplicación. Los tratamientos se aplicaron en una ocasión en cada estación lluviosa en las mismas parcelas experimentales. Se determinó la densidad de población de malezas antes de la aplicación de los tratamientos y se evaluó el control de malezas y la producción y calidad del forraje a los 42, 98, 160 y 223 días después de la aplicación (DDA) en el primer período de lluvias y a los 34, 72, 112 y 163 DDA en el segundo. Los tratamientos con herbicidas tuvieron un mayor control de malezas que el chapeo con machete, lo cual se reflejó en este último tratamiento en producciones de forraje de 17,4 a $66,6 \%$ menores que el promedio obtenido en los tratamientos herbicidas. En las épocas de menor humedad, el contenido de proteína cruda del pasto Llanero fue significativamente mayor, al menos en uno de los tratamientos herbicidas, en relación al testigo sin aplicación.
\end{abstract}

Palabras clave: Pastizales, herbicidas, chapeo, proteína cruda, fibra detergente neutro.

\begin{abstract}
Weed control in the productivity and quality of Gamba grass. From August 2003 to December 2004 a field test was carried out during two rainy seasons in the Municipality of Cotaxtla, Veracruz, Mexico, in order to determine the effect of different treatments to control weeds on the production and quality of Gamba grass. A completely randomized block design with four replications was used. The treatments were: 1 . Picloram (4-amino-3,5,6-trichloro-2pyridinecarboxylic acid) + fluroxypir (4-amino-3,5-dichloro6-fluoro-2-pyridyloxyacetic acid) $(40 \mathrm{~g}+40 \mathrm{~g} / 1001$ water), 2. Picloram + 2,4-D (2,4-dichlorophenoxyacetic acid) (64 g $+240 \mathrm{~g} / 1001$ water) 3 . Mowing with machete, and 4 . Weedy check. The treatments were applied once per rainy season in the same experimental plots. Weed population density was determined before treatment application, whereas weed control and forage production and quality were evaluated at 42, 98, 160 and 223 days after application (DAA) during the first rainy season and at 34, 72, 112 and 163 DAA during the second one. The two herbicide treatments resulted in a higher weed control than the mowing treatment, which was reflected in the last treatment in a reduction of the forage production 17,4 to $66,6 \%$ lower than the average obtained with the herbicide treatments. During the lowest moisture periods, the crude protein content of the Gamba grass was significantly higher at least in one of the herbicide treatments in relation with the weedy check.
\end{abstract}

Key words: Grasslands, herbicides, mowing, crude protein, neutral detergent fiber.

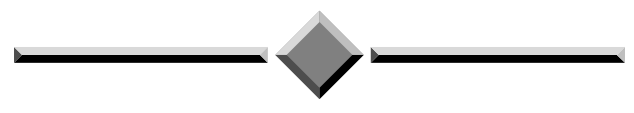

\footnotetext{
1 Recibido: 25 de abril, 2009. Aceptado: 17 de mayo, 2010. Convenio de Investigación INIFAP-Dow AgroSciences S. A. de C. V.

2 Campo Experimental Cotaxtla. CIRGOC. Instituto Nacional de Investigaciones Agrícolas y Pecuarias (INIFAP). Km. 34 Carr. VeracruzCórdoba, mpio. de Medellín de Bravo, Veracruz, México. esqueda.valentin@inifap.gob.mx

3 Campo Experimental La Posta. CIRGOC. Instituto Nacional de Investigaciones Agrícolas y Pecuarias (INIFAP). Km 22.5 Carr. VeracruzCórdoba, Col. Paso del Toro, mpio. de Medellín de Bravo, Veracruz, México. montero.maribel@inifap.gob.mx

4 Facultad de Medicina Veterinaria y Zootecnia. Universidad Veracruzana. Veracruz, Veracruz, México. fjuarez@uv.mx
} 


\section{INTRODUCCIÓN}

En los potreros de las regiones tropicales del estado de Veracruz, se presentan principalmente malezas dicotiledóneas, entre las cuales se pueden tener especies herbáceas, semileñosas y leñosas (Guevara et al. 1994, Enríquez et al. 1999, Esqueda et al. 2005). Si éstas no se controlan con oportunidad y eficiencia, reducen la producción y calidad del forraje, incrementan los costos del manejo y producción del ganado, dificultan el pastoreo, reducen la tasa de reproducción, la ganancia de peso de los animales y algunas pueden ser tóxicas (Panter y James 1990, Miller 1992, DiTomaso 2000, Grice y Campbell 2000, Carmona et al. 2001, Pellegrini et al. 2007). De hecho, las malezas pueden ocasionar más pérdidas económicas en los pastizales que todas las demás plagas en conjunto (Bovey 1987), por lo que un manejo adecuado de las mismas, se refleja en alta producción de forraje, menor tiempo de engorde del ganado y por lo tanto, en mayores ganancias para el productor (Esqueda 2003).

Los principales métodos para controlar malezas en los potreros tropicales, son los chapeos manuales o mecánicos y la aplicación de herbicidas selectivos del tipo hormonal (Vitelli 2000, Esqueda 2003). Los chapeos se recomiendan para superficies pequeñas (Sheley et al. 1998) y solamente controlan las malezas por tiempo reducido, ya que producen podas, que eliminan la dominancia apical de la planta y estimulan las yemas laterales del tallo, lo que agrava el problema de infestación (Sosa y Medrano 1992). Por su parte, con la aplicación de herbicidas reguladores del crecimiento, se reducen significativamente las poblaciones de malezas de hoja ancha (Motooka 1986), lo cual repercute en un aumento significativo en la producción de forraje (Esqueda y Tosquy 2007).

La mezcla de picloram + 2,4-D, desarrollada en la década de los 60's (Timmons 1970) ha sido por muchos años el principal tratamiento para el control químico de malezas en pastizales; sin embargo, su efecto sobre algunas especies es irregular o no eficiente (Esqueda et al. 2005). El fluroxipir es un herbicida hormonal, que penetra y se mueve rápidamente por el interior de las plantas (Sanders y Pallet 1987), por lo que en mezcla con el picloram, puede ser una alternativa para el control químico de malezas en potreros y pastizales, debido a que su actividad es mayor que la del 2,4-D. En relación con lo anterior, en estudios realizados en potreros tropicales del estado de Veracruz, la mezcla de picloram + fluroxipir mostró una efectividad semejante o mayor que la de picloram $+2,4$-D para controlar diferentes especies de malezas perennes (Esqueda 2001, Esqueda et al. 2005).

Aunque se ha indicado que el control químico de las malezas es más eficiente que el chapeo (Carmona et al. 2001), en México existen pocos estudios en que se haya comparado la producción y calidad de forraje de pastos tropicales, obtenida al controlar las malezas con ambos tratamientos. Para los pastos Pangola (Digitaria decumbens Stent) y Estrella de África [Cynodon plectostachyus (K. Schum.) Pilger] se determinó que el control de las malezas con herbicidas fue significativamente mayor que el chapeo con machete, lo que influyó en la reducción del rendimiento de forraje seco en este último tratamiento, entre 10,3 y $47 \%$ para Pangola (Esqueda y Tosquy 2007) y entre 27,6 y 58\%, para Estrella de África (Esqueda et al. 2009). Además, el porcentaje de proteína cruda en este último pasto, fue mayor en periodos de escasez de humedad bajo el control de las malezas con herbicidas, que cuando se utilizó el chapeo con machete (Esqueda et al. 2009).

El objetivo del presente trabajo fue determinar el efecto del método de combate de malezas en la productividad y calidad del pasto Llanero.

\section{MATERIALES Y MÉTODOS}

De agosto de 2003 a diciembre de 2004 se condujo un experimento en la localidad de San Ramón, ubicada en el municipio de Cotaxtla, Veracruz, México, en un potrero con pasto Llanero (Andropogon gayanus Kunth), el cual fue rodeado con una cerca electrificada para controlar el acceso del ganado. El potrero se seleccionó por tener una alta cobertura de malezas $(68,6 \%)$. El clima de la localidad es Aw" ${ }_{1}(w)$ eg, que corresponde a un subtipo de humedad media dentro de los cálidos subhúmedos (García 1987).

Se evaluaron cuatro tratamientos en un diseño experimental de bloques al azar con cuatro repeticiones: 1. Picloram + fluroxipir $(40 \mathrm{~g}+40 \mathrm{~g}$ en $1001 \mathrm{de}$ agua $)$, 2. Picloram + 2,4-D (64 g $+240 \mathrm{~g}$ en 1001 de agua), 3. Chapeo con machete y 4 . Testigo sin aplicación. Las dimensiones de las parcelas experimentales fueron de 22,50 m de longitud x 13,30 m de ancho. Cada tratamiento se aplicó durante dos periodos en una ocasión por ciclo en las mismas parcelas experimentales. En el primero, se efectuó el 21 de agosto de 2003, cuando las 
malezas tuvieron una altura de entre 25 y $90 \mathrm{~cm}$, y el segundo, el 30 de junio de 2004, cuando la altura varió entre 10 y $80 \mathrm{~cm}$. Se empleó una aspersora manual de mochila, equipada con una boquilla Tee Jet 8003 VP. La aplicación se efectuó en forma dirigida a las malezas, cubriéndolas completamente con la solución aplicada, pero sin llegar al escurrimiento. En el primer ciclo se adicionó en promedio 73,20 litros de solución en las cuatro parcelas de cada tratamiento de control químico, mientras que en el segundo, debido a la reducción en la población ocasionada por los herbicidas aplicados el ciclo anterior, sólo se necesitaron 26,24 litros. El lote experimental se desarrolló bajo condiciones de temporal y no se fertilizó, ni fue necesario aplicar insecticidas. En el primer periodo se tuvo una precipitación pluvial de $547 \mathrm{~mm}$, mientras que en el segundo periodo, la precipitación pluvial fue de 488,5 mm (Cuadro 1).

Cuadro 1. Distribución mensual de la precipitación pluvial en el periodo de agosto de 2003 a diciembre de 2004. San Ramón, mpio. de Cotaxtla, Veracruz, México.

\begin{tabular}{lccc}
\hline $\begin{array}{c}\text { Mes y año } \\
\text { (Periodo 1) }\end{array}$ & $\begin{array}{c}\text { Precipita- } \\
\text { ción }(\mathbf{m m})\end{array}$ & $\begin{array}{c}\text { Mes y año } \\
\text { (Periodo 2) }\end{array}$ & $\begin{array}{c}\text { Precipita- } \\
\text { ción }(\mathbf{m m})\end{array}$ \\
\hline Agosto 2003 & 83,5 & Junio 2004 & 143,0 \\
Septiembre 2003 & 295,0 & Julio 2004 & 95,0 \\
Octubre 2003 & 122,0 & Agosto 2004 & 103,0 \\
Noviembre 2003 & 25,0 & Septiembre 2004 & 27,5 \\
Diciembre 2003 & 0,0 & Octubre 2004 & 109,0 \\
Enero 2004 & 9,5 & Noviembre 2004 & 4,0 \\
Febrero 2004 & 12,0 & Diciembre 2004 & 7,0 \\
Marzo 2004 & 0,0 & & \\
\hline
\end{tabular}

\section{Densidad de población de malezas}

La densidad de población de malezas se obtuvo una vez por periodo, antes de la aplicación de los tratamientos, mediante conteos por especie en el interior de un cuadro de $2 \times 2 \mathrm{~m}$, colocado al azar en cada una de las parcelas experimentales, como lo indican Burrill et al. (1977) y Harker et al. (2000). En el primer periodo, las densidades se obtuvieron al promediar los datos de cada especie en las 16 parcelas experimentales, mientras que en el segundo, al existir diferencias por efecto de los tratamientos del primer período, se determinaron promediando los datos por especie en las cuatro parcelas de cada tratamiento. Para la identificación de las malezas, se colectaron tres ejemplares de cada especie presente y se enviaron al Laboratorio de Etnobotánica del Programa de Botánica del Colegio de Postgraduados en Ciencias Agrícolas, en Montecillo, Edo. de México.

\section{Control de malezas}

Se evaluó visualmente el efecto de tratamientos en el control de Pachecoa prismatica (Sessé y Moc.) Standl. \& B. G. Schub., que fue la especie dominante, mientras que las demás especies de malezas semiarbustivas y herbáceas, fueron evaluadas como un solo grupo. La evaluación se realizó mediante la comparación visual de las parcelas tratadas con las no tratadas, de acuerdo a Enloe et al. (2008) y DiTomaso et al. (2008), siendo éste, el método de evaluación más utilizado internacionalmente para cuantificar el control de malezas (Burrill et al. 1977, Frans et al. 1986). Se utilizó la escala porcentual (0 a 100\%), en donde 0 significó que las malezas no fueron afectadas y $100 \%$, que fueron completamente eliminadas (Alemán 2004). En el primer periodo, las evaluaciones se realizaron a los 42, 98, 160 y 223 días después de la aplicación de los tratamientos (DDA), mientras que en el segundo período, ésto se realizó a los 34, 72, 112 y 163 DDA. Las fechas de evaluación se ajustaron a la toma de muestras para producción y calidad del pasto, en la etapa vegetativa antes de la floración.

\section{Producción de materia seca del pasto Llanero}

Para estimar la producción de materia seca del pasto Llanero, en cada época de muestreo, se lanzó al azar en cuatro ocasiones un cuadro de 1 x $1 \mathrm{~m}$ en cada parcela experimental, y se cortó con machete la parte aérea de las plantas de pasto del interior, a una altura de $10 \mathrm{~cm}$ por arriba del nivel del suelo (Middleton 1982) (Penati et al. 2005). El forraje de los cuatro cuadros de cada parcela experimental, se pesó en el sitio experimental con una báscula de reloj Cor No. 5394, se mezcló y se tomó una muestra de $200 \mathrm{~g}$, la cual se colocó en una estufa de aire forzado RIOSSA modelo H-41 a 100 ${ }^{\circ} \mathrm{C}$ por 48 horas y su peso seco se utilizó para calcular 
el porcentaje de materia seca de forraje por parcela. Después de cada muestreo, se permitió el acceso del ganado al lote experimental por tres días, para consumir el pasto disponible y provocar su rebrote.

\section{Calidad del forraje del pasto Llanero}

Para la determinación de la calidad del forraje se colectaron cinco muestras de pasto fresco de cada parcela experimental (un muestreo en cada esquina y uno en el centro de la parcela), se mezclaron y se tomó una muestra de $500 \mathrm{~g}$, la cual se llevó al Laboratorio de Nutrición Animal y Forrajes del Campo Experimental La Posta del INIFAP, en donde se le realizaron los análisis bromatológicos correspondientes. Las muestras se colocaron en una estufa de aire forzado RIOSSA modelo $\mathrm{H}-41$ a $55^{\circ} \mathrm{C}$ para secar a peso constante y se molieron en un molino Wiley con malla de $1 \mathrm{~mm}$ para realizar los siguientes análisis, aprobados por la Asociación Oficial de Químicos Analíticos (AOAC 1990): 1. Determinación de proteína cruda por el procedimiento de Macro Kjeldahl, 2. Determinación de materia orgánica por diferencia entre la materia seca y las cenizas, y 3. Determinación de fibra detergente neutro por el procedimiento de Goering y Van Soest (1970). La producción de proteína cruda por hectárea se calculó multiplicando la producción de forraje seco de cada tratamiento por su porcentaje de proteína cruda.

\section{Análisis estadísticos}

Se realizaron análisis de varianza de los datos experimentales, y como prueba de separación de promedios se utilizó Tukey $(\alpha=0,05)$. Antes de realizar los análisis, y con la finalidad de homogenizar las varianzas, los datos de control de malezas (expresados en porcentaje), fueron transformados a su valor de arco seno como lo recomiendan Gomez y Gomez (1984) y Frans et al. (1986).

\section{RESULTADOS Y DISCUSIÓN}

\section{Periodo de agosto de 2003 a marzo de 2004}

Densidad de población de malezas. Antes de la aplicación de los tratamientos se cuantificó una densidad de población de malezas equivalente a 110000 plantas/ha, compuesta por 13 especies de siete familias. La Asteraceae y Malvaceae aportaron el mayor número de especies con tres, cada una (Cuadro 2). La especie dominante fue $P$. prismatica, un arbusto perenne de la familia Fabaceae, que se encuentra en algunas zonas tropicales de México, Centroamérica y el norte de Sudamérica (Norman y Gunn 1985); aunque no fue la especie con mayor población, debido a su tamaño y desarrollo, su cobertura representaba alrededor del $60 \%$ de la cobertura total de malezas. Las otras especies semiarbustivas y herbáceas, son comúnmente encontradas en las zonas tropicales de México (Villaseñor y Espinosa 1998).

Control de $\boldsymbol{P}$. prismatica. La mezcla de picloram + fluroxipir proporcionó un control de esta especie superior al $99 \%$ en todas las épocas de evaluación; a su vez, picloram + 2,4-D tuvo un control total hasta los 98 DDA y al final, éste fue ligeramente inferior a 99\%. En todas las épocas de evaluación, los controles obtenidos con ambos tratamientos herbicidas, fueron estadísticamente semejantes entre sí, y superiores a los obtenidos con el chapeo manual, cuyo control a los 42 DDA fue de $86,3 \%$, y se redujo paulatinamente hasta terminar en $73,8 \%$ a los 223 DDA (Cuadro 3). Lo anterior, coincide con lo indicado por Pinzón y Montenegro (1988) en el control de malezas en pasto Jaragua (Hyparrhenia rufa) en Panamá y por Valbuena y Acosta (2006) en pasto Estrella (Cynodon nlemfuensis) en Venezuela. El menor control de esta especie ofrecido por el chapeo con machete se debe a la capacidad que tienen los arbustos de formar nuevas ramas después de efectuada esta práctica (Scanlan 1984), mientras que los herbicidas de tipo hormonal, al ser translocables (Thomson 1993), impiden o reducen en gran medida la emisión de rebrotes, por lo que pueden ofrecer buenos controles de este tipo de malezas por varios meses (Enloe et al. 2008, Samuel y Lym 2008).

Control de malezas de hoja ancha semiarbustivas y herbáceas. Los dos tratamientos herbicidas fueron similares estadísticamente en el combate del complejo de malezas herbáceas y semi arbustivas en todas las épocas de evaluación. El control inicial con picloram + fluroxipir fue cercano a $98 \%$ y se redujo cerca de $93 \%$ a los 223 DDA; a su vez, el control obtenido con picloram + 2,4-D fue ligeramente menor, y llegó a estar por debajo del 90\% a los 160 DDA, aunque se incrementó ligeramente al final. Los dos tratamientos herbicidas superaron ampliamente el control de malezas herbáceas obtenido con el chapeo, que en la primera evaluación fue menor a $70 \%$ y terminó en 
Cuadro 2. Densidad de población de malezas en pasto Llanero (Andropogon gayanus) al inicio del experimento, en agosto de 2003. San Ramón, municipio de Cotaxtla, Veracruz, México.

\begin{tabular}{lccc}
\hline \multicolumn{1}{c}{ Nombre científico } & Familia & Tipo de crecimiento & Número plantas/ha \\
\hline Croton hirtus L'Her. & Euphorbiaceae & Herbáceo & 18750 \\
Mimosa pudica L. & Mimosaceae & Semiarbustivo & 15350 \\
Croton sp. & Euphorbiaceae & Herbáceo & 12500 \\
Sida acuta Burm. f. & Malvaceae & Herbáceo & 11875 \\
Malachra alceifolia Jacq. & Malvaceae & Herbáceo & 10625 \\
Pachecoa prismatica (Sessé y Moc.) & & & \\
Standl. \& B.G. Schub. & Fabaceae & Arbustivo & 10500 \\
Tamonea curassavica (L.) Pers. & Verbenaceae & Herbáceo & 9700 \\
Senna obtusifolia (L.) Irwin \& Barneby & Caesalpiniaceae & Semiarbustivo & 7250 \\
Sida spinosa L. & Malvaceae & Herbáceo & 5900 \\
Aeschynomene americana L. & Fabaceae & Semiarbustivo & 4700 \\
Lagascea mollis Cav. & Asteraceae & Herbáceo & 1250 \\
Melampodium americanum L. & Asteraceae & Herbáceo & 950 \\
Baltimora recta L. & Asteraceae & Herbáceo & 650 \\
\hline Total & & & 110000 \\
\hline
\end{tabular}

33,8\% (Cuadro 3). Cabe señalar, que la eliminación de $P$. prismatica mediante el chapeo, permitió mayor emergencia o desarrollo de las especies de malezas no dominantes, que al crecer bajo la sombra de la especie arbustiva, presentaban un crecimiento raquítico, situación que ha sido también indicada al eliminar la especie arbustiva Mimosa albida en potreros del norte del estado de Veracruz (Esqueda y Tosquy 2007).
Producción de materia seca del pasto Llanero. En la primera época de evaluación, los dos tratamientos de control químico permitieron una producción de materia seca promedio cercana a los $3500 \mathrm{~kg} / \mathrm{ha}$, que fue estadísticamente superior a la que se obtuvo con el chapeo con machete y a la del testigo sin aplicación, con los que sólo produjeron 67 y $24 \%$, respectivamente, del promedio de la materia seca obtenida con

Cuadro 3. Control de las malezas (\%) P. prismatica y semiarbustivas y herbáceas a días después de la aplicación de los tratamientos en pasto Llanero (Andropogon gayanus). Período de agosto de 2003 a marzo de 2004. San Ramón, municipio de Cotaxtla, Veracruz, México.

\begin{tabular}{|c|c|c|c|c|c|c|c|c|}
\hline \multirow[t]{3}{*}{ Tratamiento } & \multicolumn{4}{|c|}{$P$. prismatica } & \multicolumn{4}{|c|}{ Malezas semiarbustivas y herbáceas } \\
\hline & \multicolumn{4}{|c|}{ Días después de la aplicación de los tratamientos } & \multicolumn{4}{|c|}{ Días después de la aplicación de los tratamientos } \\
\hline & 42 & 98 & 160 & 223 & 42 & 98 & 160 & 223 \\
\hline Picloram + fluroxipir & 99,8 a & 99,5 a & 99,8 a & 99,8 a & 97,8 a & 95,8 a & $93,0 \mathrm{a}$ & 92,8 a \\
\hline Picloram + 2,4-D & $100,0 \mathrm{a}$ & $10,0 \mathrm{a}$ & $98,5 \mathrm{a}$ & 98,8 a & $94,3 \mathrm{a}$ & 92,8 a & 87,8 a & $91,0 \mathrm{a}$ \\
\hline Control con machete & $86,3 \mathrm{~b}$ & $80,0 \mathrm{~b}$ & $73,8 \mathrm{~b}$ & $73,8 \mathrm{~b}$ & $67,5 \mathrm{~b}$ & $50,0 \mathrm{~b}$ & $36,3 \mathrm{~b}$ & $33,8 \mathrm{~b}$ \\
\hline Testigo sin aplicación & $0,0 \mathrm{c}$ & $0,0 \mathrm{c}$ & $0,0 \mathrm{c}$ & $0,0 \mathrm{c}$ & $0,0 \mathrm{c}$ & $0,0 \mathrm{c}$ & $0,0 \mathrm{c}$ & $0,0 \mathrm{c}$ \\
\hline
\end{tabular}

Valores con la misma letra en una misma columna, son semejantes (Tukey $\alpha=0,05$ ). Las comparaciones son entre tratamientos en cada época de evaluación. 
los tratamientos de control químico. A su vez, con el chapeo se produjo 2,76 veces más materia seca, que en el testigo sin aplicar. En todos los tratamientos, se observó una reducción en la producción de materia seca entre una época de evaluación y la siguiente, debido a la paulatina disminución de la humedad del terreno, ya que los muestreos dos, tres y cuatro, se realizaron en los meses de noviembre a marzo, cuando la precipitación pluvial fue escasa o nula. A los 98 DDA, la producción de materia seca con el tratamiento a base de picloram + fluroxipir fue mayor a la de picloram $+2,4-$ $\mathrm{D}$, si bien, no se detectó diferencia estadística. También, fueron semejantes las producciones obtenidas con la aplicación de picloram + 2,4-D y con el chapeo, aunque, con la aplicación de la mezcla herbicida, se produjo $42 \%$ más materia seca. Para esta evaluación, la producción del testigo sin aplicación fue estadísticamente semejante a la del control con machete. En la tercera y cuarta evaluación, las dos mezclas herbicidas produjeron una cantidad semejante de materia seca de pasto, superando estadísticamente al chapeo y al testigo sin aplicación, cuyas producciones no fueron significativamente diferentes entre sí (Figura 1).

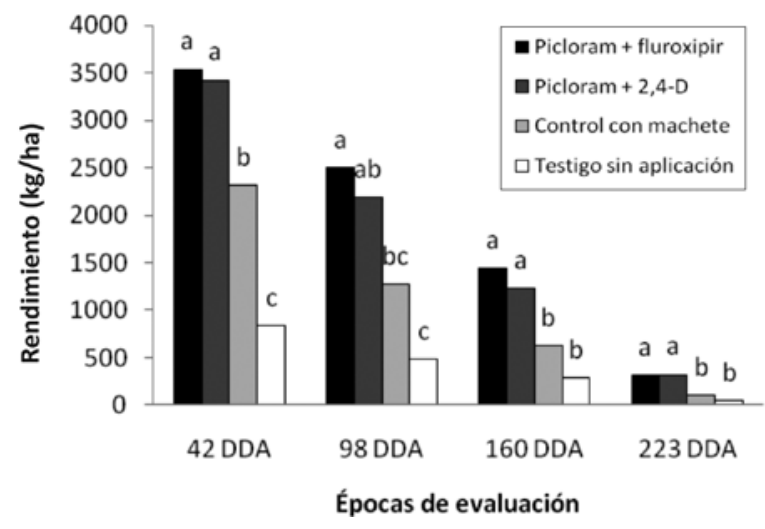

Figura 1. Rendimiento de materia seca de forraje de pasto Llanero (Andropogon gayanus) (kg/ha) a diferentes días después de la aplicación de los tratamientos (DDA). Período de agosto de 2003 a marzo de 2004. San Ramón, mpio. de Cotaxtla, Veracruz, México.

La obtención de mayores producciones de materia seca de pasto, debido a un control de malezas más eficiente cuando se aplican herbicidas, con respecto al chapeo, concuerda con lo que se ha indicado en experimentos establecidos en Veracruz, con los pastos Pangola (Esqueda y Tosquy 2007) y Estrella de África (Esqueda et al. 2009). De acuerdo a estos resultados, al controlar las malezas con chapeos, la producción de forraje, permite mantener en el potrero una carga animal entre dos y tres veces mayor que la que soportaría un potrero sin control de malezas, mientras que si se aplican herbicidas, la carga animal se podría incrementar entre cuatro y seis veces, lo cual maximiza la producción de carne o leche (Valbuena y Acosta 2006).

Proteína cruda. En los dos primeros cortes, el contenido de proteína cruda en el pasto de todos los tratamientos fue estadísticamente semejante. A los 160 DDA, el pasto del testigo sin aplicación y del tratamiento de control con machete, tuvo significativamente menor cantidad de proteína cruda que aquellos en que las malezas fueron controladas con picloram + 2,4-D, pero su contenido fue semejante a los del tratamiento de picloram + fluroxipir. A los 223 DDA la cantidad de proteína cruda fue semejante en los pastos de todos los tratamientos, aunque estos resultados pueden considerarse atípicos, ya que la escasa precipitación recibida desde el muestreo anterior, ocasionó la existencia de escaso material vegetativo, especialmente en las parcelas del testigo sin aplicación. En estas parcelas, a consecuencia del pastoreo, el pasto casi desapareció, por lo que se muestrearon rebrotes muy tiernos y con un alto contenido de proteína cruda, característica normal en pastos tropicales jóvenes (Juárez et al. 2005) (Cuadro 4).

Aunque no se detectaron diferencias concluyentes en la concentración de proteína cruda en los pastos por efecto de los tratamientos, al calcular su producción por hectárea, se observó que el control químico superó ampliamente al testigo sin aplicación y en menor proporción al control con machete, sobre todo en los dos últimos muestreos, que coincidieron con la menor precipitación pluvial.

Materia orgánica. El contenido de materia orgánica del pasto varió entre 89,4 y $93,8 \%$, y no fue afectado por los métodos de control de malezas en ninguna de las épocas de evaluación (datos no presentados).

Fibra detergente neutro. A los 42 DDA, la mayor concentración de fibra detergente neutro se encontró en los pastos del testigo sin aplicación, siendo semejante a la del control con machete, que a su vez, fue estadísticamente similar a la de los dos tratamientos 
Cuadro 4. Efecto de los tratamientos en el contenido y producción de proteína cruda del pasto Llanero (Andropogon gayanus) días después de la aplicación de los tratamientos. Período de agosto de 2003 a marzo de 2004. San Ramón, mpio. de Cotaxtla, Veracruz, México.

\begin{tabular}{|c|c|c|c|c|c|c|c|c|}
\hline \multirow[t]{3}{*}{ Tratamiento } & \multicolumn{4}{|c|}{ Proteína cruda (\%) } & \multicolumn{4}{|c|}{ Proteína cruda (kg/ha) } \\
\hline & \multicolumn{4}{|c|}{$\begin{array}{c}\text { Días después de la aplicación de los } \\
\text { tratamientos }\end{array}$} & \multicolumn{4}{|c|}{$\begin{array}{c}\text { Días después de la aplicación de los } \\
\text { tratamientos }\end{array}$} \\
\hline & 42 & 98 & 160 & 223 & 42 & 98 & 160 & 223 \\
\hline Picloram + fluroxipir & $8,2 \mathrm{a}^{*}$ & $7,0 \mathrm{a}$ & $6,8 \mathrm{ab}$ & $7,7 \mathrm{a}$ & $292 \mathrm{a}$ & $173 \mathrm{a}$ & $98 \mathrm{a}$ & $24 \mathrm{a}$ \\
\hline Picloram + 2,4-D & 8,1 a & $6,7 \mathrm{a}$ & $7,4 \mathrm{a}$ & $8,4 \mathrm{a}$ & $276 \mathrm{ab}$ & $146 \mathrm{ab}$ & $92 \mathrm{a}$ & $27 \mathrm{a}$ \\
\hline Control con machete & $8,5 \mathrm{a}$ & $7,0 \mathrm{a}$ & $5,9 \mathrm{~b}$ & $7,9 \mathrm{a}$ & $195 \mathrm{~b}$ & $90 \mathrm{bc}$ & $36 \mathrm{~b}$ & $8 \mathrm{~b}$ \\
\hline Testigo sin aplicación & $7,2 \mathrm{a}$ & $6,1 \mathrm{a}$ & $6,0 \mathrm{~b}$ & $8,3 \mathrm{a}$ & $61 \mathrm{c}$ & $31 \mathrm{c}$ & $17 \mathrm{~b}$ & $4 b$ \\
\hline
\end{tabular}

* Valores con la misma letra en una misma columna, son semejantes (Tukey $\alpha=0,05$ ). Las comparaciones son entre tratamientos en cada época de evaluación.

de control químico. Sin embargo, esta situación no se observó a los 98 DDA en donde no se presentaron diferencias significativas entre tratamientos para esta variable. Sin embargo, a los 160 DDA, se volvió a observar un mayor contenido de este componente en el tratamiento de control con machete y el testigo sin aplicar. Como se indicó para proteína cruda, el resultado del último muestreo es atípico, por las condiciones indicadas que favorecieron los valores menores que se observaron en el testigo sin aplicar (Cuadro 5).

\section{Resultados del período de junio a diciembre de 2004}

Densidad de población de malezas. Al inicio del período de lluvias 2004, la población de malezas del testigo sin aplicación fue de 61875 plantas/ha; en donde $P$. prismatica se mantuvo como la especie dominante y su altura varió entre 60 y $140 \mathrm{~cm}$. Debido al chapeo efectuado en 2003, en las parcelas correspondientes a este tratamiento, se redujo la población de esta especie a 41875 plantas/ha, y su altura fue menor (40 a $80 \mathrm{~cm}$ ), aunque también fue la especie más importante. En las parcelas aplicadas el período anterior con picloram + 2,4-D y picloram + fluroxipir, se observó una fuerte reducción en la densidad y composición de las poblaciones de malezas, ya que no existieron plantas de $P$. prismatica, y las poblaciones de las otras especies, fueron en promedio 66,4 y 77,3\% más bajas que las del tratamiento de chapeo y el testigo sin aplicación, respectivamente. Además, en relación con las especies encontradas en el primer muestreo se detectó la presencia de Hyptis suaveolens y no se encontró en esta ocasión Aeschynomene americana (Cuadro 6).

Cuadro 5. Efecto de los tratamientos en el contenido de fibra detergente neutro (\%) del pasto Llanero (Andropogon gayanus) días de la aplicación de los tratamientos (DDA). Período de agosto de 2003 a marzo de 2004. San Ramón, mpio. de Cotaxtla, Veracruz, México.

\begin{tabular}{lcccc}
\hline & \multicolumn{4}{c}{ Días después de la aplicación de los tratamientos } \\
\cline { 2 - 5 } \multicolumn{1}{c}{ Tratamiento } & $\mathbf{4 2}$ & $\mathbf{9 8}$ & $\mathbf{1 6 0}$ & $\mathbf{2 2 3}$ \\
\hline Picloram + fluroxipir & $64,4 \mathrm{~b} *$ & $65,9 \mathrm{a}$ & $63,9 \mathrm{ab}$ & $65,1 \mathrm{a}$ \\
Picloram + 2,4-D & $64,7 \mathrm{~b}$ & $66,9 \mathrm{a}$ & $61,8 \mathrm{~b}$ & $64,3 \mathrm{ab}$ \\
Control con machete & $65,9 \mathrm{ab}$ & $67,0 \mathrm{a}$ & $65,7 \mathrm{a}$ & $65,1 \mathrm{a}$ \\
Testigo sin aplicación & $66,6 \mathrm{a}$ & $66,4 \mathrm{a}$ & $64,6 \mathrm{a}$ & $62,4 \mathrm{~b}$ \\
\hline
\end{tabular}

* Valores con la misma letra en una misma columna, son semejantes (Tukey $\alpha=0,05$ ). Las comparaciones son entre tratamientos en cada época de evaluación. 
Cuadro 6. Densidad de población de malezas (plantas/ha) presentes en pasto Llanero (Andropogon gayanus) al inicio del período 2004. San Ramón, municipio de Cotaxtla, Veracruz, México.

\begin{tabular}{lrrrr}
\hline Nombre científico & $\begin{array}{c}\text { Picloram + } \\
\text { fluroxipir }\end{array}$ & Picloram + 2,4-D & Chapeo con machete & $\begin{array}{c}\text { Testigo } \\
\text { sin licación }\end{array}$ \\
\hline Croton hirtus & 1250 & 1250 & 7500 & 1250 \\
Mimosa pudica & 1250 & 3125 & 11875 & 16250 \\
Croton sp. & 0 & 625 & 625 & 0 \\
Sida acuta & 6250 & 4375 & 8125 & 4375 \\
Malachra alceifolia & 0 & 0 & 0 & 2500 \\
Pachecoa prismatica & 0 & 0 & 4375 & 10625 \\
Tamonea curassavica & 1250 & 625 & 5625 & 10000 \\
Senna obtusifolia & 0 & 625 & 0 & 0 \\
Sida spinosa & 0 & 0 & 2500 & 0 \\
Hyptis suaveolens (L.) Poit & 1250 & 625 & 0 & 1250 \\
Lagascea mollis & 1250 & 1250 & 0 & 12500 \\
Melampodium americanum & 1250 & 1875 & 1250 & 2500 \\
Baltimora recta & 0 & 0 & 0 & 625 \\
\hline Total & 13750 & 14375 & 815 & 61875 \\
\hline
\end{tabular}

Debido a la reducción en la densidad de población en las parcelas aplicadas en 2003 con herbicidas, en el segundo periodo solamente se necesitó aplicar el $35,8 \%$ de la solución herbicida adicionada en el primer período de lluvias. En trabajos semejantes realizados con otros pastos en el estado de Veracruz, se han observado reducciones de $95,3 \%$ en las poblaciones de M. albida (Esqueda y Tosquy 2007) y de 78,4\% de Sida rhombifolia (Esqueda et al. 2009), al inicio del período de lluvias posterior al que se aplicaron herbicidas para su control, y la cantidad necesaria para el segundo periodo fue solamente 17,9 y $67,1 \%$ de la original. El efecto de los herbicidas aplicados en un ciclo, sobre la reducción en la población malezas, en el siguiente, también lo han indicado Koger et al. (2006), para Lespedeza cuneata en pastizales de Oklahoma, y Samuel y Lym (2008) para Cirsium arvense en Dakota del Norte.

Control de $P$. prismatica. La mezcla de picloram + fluroxipir tuvo un control total de P. prismatica hasta los 112 DDA, el cual se redujo a $98,8 \%$ a los 163 DDA. El picloram + 2,4-D, controló completamente a esta especie hasta la última época de evaluación, en el control obtenido con los dos tratamientos a base de herbicidas fue estadísticamente semejante. El chapeo con machete tuvo un buen control inicial $(83,8 \%)$, pero este efecto fue solamente temporal y disminuyó entre épocas de evaluación, para terminar con un control ligeramente superior al $60 \%$. En todas las épocas de evaluación, el control de $P$. prismatica obtenido con los tratamientos de picloram + fluroxipir y picloram $+2,4$-D fue significativamente superior al del control con machete (Cuadro 7).

Es importante recalcar, que al no existir plantas de $P$. prismatica en las parcelas correspondientes a los dos tratamientos de control químico al momento de la aplicación, el control de esta especie en el segundo período, puede atribuirse en gran medida a la aplicación realizada al inicio del primer período, lo cual coincide en gran medida con lo indicado en otros trabajos de investigación para M. albida (Esqueda y Tosquy 2007) y S. rhombifolia (Esqueda et al. 2009).

Control de malezas de hoja ancha semiarbustivas y herbáceas. En las diferentes épocas de evaluación, la mezcla de picloram + fluroxipir tuvo un control de las especies de malezas semiarbustivas y herbáceas de entre 95 y $98 \%$, mientras que el de picloram + 2,4-D varió entre 91,8 y 95,5\%. Sin embargo, en términos estadísticos, ambos tratamientos tuvieron controles semejantes. Por otra parte, el chapeo con machete tuvo un efecto limitado, ya que desde la evaluación de los 34 DDA, su control sólo fue ligeramente superior a $60 \%$, para terminar por debajo de $50 \%$ a los 163 DDA y en todos los casos, su control fue inferior 
Cuadro 7. Control de P. prismatica y malezas semiarbustivas y herbáceas (\%) a días después de la aplicación de los tratamientos (DDA) en pasto Llanero (Andropogon gayanus). Período de junio a diciembre de 2004. San Ramón, municipio de Cotaxtla, Veracruz, México.

\begin{tabular}{|c|c|c|c|c|c|c|c|c|}
\hline \multirow[t]{3}{*}{ Tratamiento } & \multicolumn{4}{|c|}{ P. prismatica } & \multicolumn{4}{|c|}{ Malezas semiarbustivas y herbáceas } \\
\hline & \multicolumn{4}{|c|}{$\begin{array}{c}\text { Días después de la aplicación de los } \\
\text { tratamientos }\end{array}$} & \multicolumn{4}{|c|}{$\begin{array}{c}\text { Días después de la aplicación de los } \\
\text { tratamientos }\end{array}$} \\
\hline & 34 & 72 & 112 & 163 & 34 & 72 & 112 & 163 \\
\hline Picloram + fluroxipir & $100,0 \mathrm{a}^{*}$ & $100,0 \mathrm{a}$ & $100,0 \mathrm{a}$ & 98,8 a & $95,0 \mathrm{a}$ & 96,3 a & $98,0 \mathrm{a}$ & $96,5 \mathrm{a}$ \\
\hline Picloram + 2,4-D & $100,0 \mathrm{a}$ & $99,8 \mathrm{a}$ & $100,0 \mathrm{a}$ & $100,0 \mathrm{a}$ & $91,8 \mathrm{a}$ & $95,3 \mathrm{a}$ & $95,5 \mathrm{a}$ & $95,5 \mathrm{a}$ \\
\hline Control con machete & $83,8 \mathrm{~b}$ & $73,8 \mathrm{~b}$ & $68,8 \mathrm{~b}$ & $61,3 \mathrm{~b}$ & $62,5 \mathrm{~b}$ & $57,5 \mathrm{~b}$ & $51,3 \mathrm{~b}$ & $47,5 \mathrm{~b}$ \\
\hline Testigo sin aplicación & $0,0 \mathrm{c}$ & $0,0 \mathrm{c}$ & $0,0 \mathrm{c}$ & $0,0 \mathrm{c}$ & $0,0 \mathrm{c}$ & $0,0 \mathrm{c}$ & $0,0 \mathrm{c}$ & $0,0 \mathrm{c}$ \\
\hline
\end{tabular}

* Valores con la misma letra en una misma columna, son semejantes (Tukey $\alpha=0,05$ ). Las comparaciones son entre tratamientos en cada época de evaluación.

al obtenido con los tratamientos a base de herbicidas (Cuadro 7).

Producción de materia seca del pasto Llanero. En el muestreo de producción de materia seca efectuado antes de la aplicación de los tratamientos en junio de 2004, se observó que la eliminación de malezas que se realizó en los tratamientos aplicados en agosto de 2003 (control químico y chapeo con machete), incrementaron la producción de forraje significativamente en comparación con la que se obtuvo en el testigo sin aplicación. Sin embargo, en las parcelas aplicadas con picloram + fluroxipir y picloram $+2,4-\mathrm{D}$, el promedio de la cantidad de materia seca producida, superó en $31,2 \%$ a la obtenida en las parcelas en que las malezas se habían chapeado con machete. Lo anterior, concuerda con lo encontrado por Esqueda et al. (2009), en un experimento similar para pasto Estrella de África, en donde la producción de materia seca fue 37,4\% mayor en los tratamientos de control químico de malezas, que en el de chapeo con machete. Después de aplicados los tratamientos en el 2004, la producción de forraje seco se incrementó en todos ellos, a excepción del testigo sin aplicación. A los 34 y 72 DDA, la mayor producción se obtuvo con picloram + fluroxipir y picloram + 2,4-D, aunque la producción de este último fue estadísticamente semejante a la obtenida con el control con machete; a su vez, en el testigo sin aplicación, la producción de materia seca de pasto aumentó ligeramente a los 72 DDA, pero fue significativamente menor que en el resto de los tratamientos. A los 112 DDA los rendimientos más altos se obtuvieron en las parcelas aplicadas con los tratamientos basados en herbicidas, si bien, la producción con picloram + fluroxipir fue estadísticamente semejante a la obtenida con machete. En esta época de evaluación se registró la producción más baja de forraje en el testigo sin aplicar. Finalmente, a los 163 DDA se observó un fuerte aumento en la cantidad de materia seca de forraje en los tratamientos herbicidas, ya que en esta época las plantas estaban maduras y su follaje tenía poca humedad, por lo que el peso seco de las cosechadas, fue en promedio el $48 \%$ del peso fresco, mientras que en las otras épocas varió entre 30 y $35 \%$. En esta última época de evaluación, la producción con picloram + fluroxipir y picloram + 2,4-D fue significativamente superior a la alcanzada con control con machete, que a su vez fue semejante a la del testigo sin aplicar (Figura 2).

En estos resultados, se mantiene la tendencia observada en el periodo anterior, al obtener mayor producción con los tratamientos de control químico que con el chapeo con machete, lo cual es indicativo de que un eficiente control de malezas es determinante para mantener un alto rendimiento de forraje en los potreros, como se ha indicado anteriormente (Peña et al. 1999, Valbuena y Acosta 2006).

Proteína cruda. En las tres primeras evaluaciones, no hubo diferencia significativa en el contenido de proteína cruda entre tratamientos y sólo a los 163 DDA, la mezcla de picloram + fluroxipir mostró un contenido estadísticamente superior al del testigo sin aplicación, pero semejante al de picloram + 2,4-D y el control con machete (Cuadro 8). 


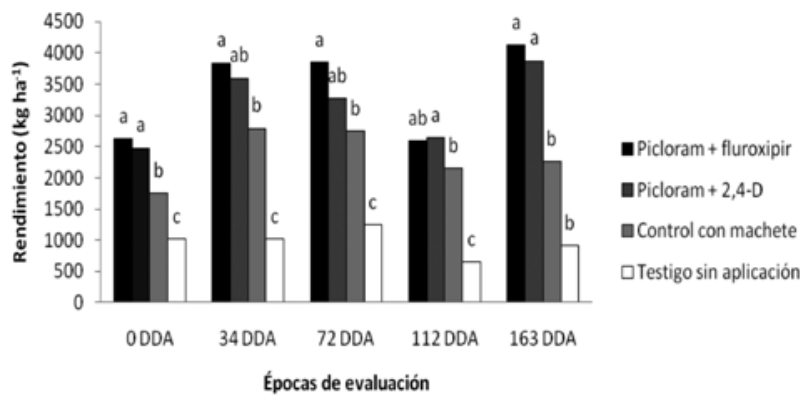

Figura 2. Rendimiento de materia seca de forraje del pasto Llanero (Andropogon gayanus) (kg/ha) a diferentes días de la aplicación de los tratamientos (DDA). Período de junio a diciembre de 2004. San Ramón, mpio. de Cotaxtla, Veracruz, México.

Los contenidos de proteína cruda a los 34 y 72 DDA, son típicos de un pasto madurado a temprana edad por la excesiva humedad y temperatura típicas de la época de verano en la zona de estudio (JuárezLagunes et al. 1999). Estas condiciones ambientales, aceleran el metabolismo de la planta, propiciando crecimiento y maduración anticipada que se asocia con un bajo contenido de proteína cruda (Contreras 2001, Rosario 2005). La concentración de proteína cruda de los pastos, está directamente relacionada con su digestibilidad, reduciéndose ésta al disminuir la proteína cruda (Van Soest 1994).

La menor temperatura y humedad a los 112 DDA permitieron una maduración más lenta y un aumento en el contenido de proteína cruda. Bajo estas condiciones favorables el valor nutritivo de los pastos fue alto, y no se apreció un efecto del control de la maleza sobre el mismo. En condiciones adversas, como el inicio de la época seca, correspondió al último muestreo, en donde hubo un efecto favorable del control químico de la maleza sobre el contenido de proteína cruda del pasto. En estas circunstancias, el pasto se encuentra en desventaja al competir contra la maleza por agua y nutrientes, por lo que a menor competencia por éstos, mayor contenido de proteína cruda (Juárez-Lagunes et al. 1999).

En un estudio con pasto Estrella de África, realizado en la parte central del estado de Veracruz, la concentración de proteína cruda, en la época seca, fue significativamente mayor en los pastos en que las malezas se controlaron con herbicidas, que en los que no se controlaron o cuyo control fue con machete (Esqueda et al. 2009).

Hasta los 112 DDA, la producción de proteína cruda/ha del tratamiento de control con machete, varió entre 82,6 y $97,6 \%$ de la obtenida en promedio con los dos tratamientos de control químico y fue estadísticamente semejante a éstos y superior a la obtenida en el testigo sin aplicación. Sin embargo, a los 163 DDA, en las parcelas con las dos mezclas herbicidas se obtuvo una producción de proteína cruda significativamente superior a la del control con machete, que fue similar a la del testigo sin aplicación (Cuadro 8).

Materia orgánica. El contenido de materia orgánica del pasto varió de 92,6 a 94\%, y al igual que en el período anterior, no hubo diferencias significativas entre tratamientos en ninguna de las épocas de evaluación (datos no presentados).

Cuadro 8. Efecto de los tratamientos en el contenido y producción de proteína cruda (PC) del pasto Llanero (Andropogon gayanus) a diferentes días después de la aplicación de los tratamientos (DDA) para el combate de malezas. Período de junio a diciembre de 2004. San Ramón, municipio de Cotaxtla, Veracruz, México.

\begin{tabular}{lcccccccc}
\hline & \multicolumn{3}{c}{ Proteína cruda $(\%)$} & \multicolumn{3}{c}{ Proteína cruda (kg/ha) } \\
\cline { 2 - 9 } & \multicolumn{3}{c}{$\begin{array}{c}\text { Días después de la aplicación de los } \\
\text { tratamientos }\end{array}$} & \multicolumn{3}{c}{ Días después de la aplicación de los } \\
tratamientos
\end{tabular}

* Valores con la misma letra en la misma columna, son semejantes (Tukey $\alpha=0,05$ ). Las comparaciones son entre tratamientos en cada época de evaluación. 
Fibra detergente neutro. El contenido de fibra detergente neutro varió entre 63,9 y 74,3\%, y fue estadísticamente semejante en todos los tratamientos en cualquiera de las épocas de evaluación (datos no presentados). Los mayores contenidos de este compuesto se tuvieron a los 163 DDA, durante la etapa con menos humedad, lo cual se debe a que el pasto tiene que invertir proteína y agua y producir fibra para resistir la presión ambiental y la competencia de las malezas. Una mayor concentración de fibra detergente neutro en los pastos, está relacionada con un menor valor nutritivo y menor consumo voluntario de forraje, por lo que el ganado puede sufrir pérdida de peso y disminución en la producción de leche y/o carne (Mertens 1994, Serna 2004).

\section{AGRADECIMIENTOS}

Se agradece a la Dra. Heike Vibrans L. ProfesoraInvestigadora del Colegio de Postgraduados en Ciencias Agrícolas su apoyo para la correcta identificación de las especies de malezas aquí mencionadas. También se agradece a la empresa Dow AgroSciences S. A. de C.V., quien financió esta investigación a través de un convenio con el Instituto Nacional de Investigaciones Forestales Agrícolas y Pecuarias.

\section{LITERATURA CITADA}

Alemán, F. 2004. Manual de investigación agronómica con énfasis en ciencia de la maleza. Imprimatur Artes Gráficas. Managua, Nicaragua. 248 p.

AOAC (Association of Official Analytical Chemists). 1990. Official methods of analysis. 15 ed. Association of Official Analytical Chemists. Arlington, VA, USA. $978 \mathrm{p}$.

Bovey, RW. 1987. Weed control problems, approaches, and opportunities in rangeland. Review Weed Science 3:57-91.

Burrill, LC; Cardenas, J; Locatelli, E. 1977. Manual de campo para investigación en control de malezas. Publicación 22-A-77. International Plant Protection Center. Oregon State University. Corvallis, OR, USA. 64 p.

Carmona, R; Carvalho, ANBS; Carvalho, PR. 2001. Controle de Acacia farnesiana e de Mimosa pteridofita em pastagem. Pesquisa Agropecuária Brasileira 36: 1301-1307.
Contreras J, JL. 2001. Determinación de la tasa de digestión de gramíneas tropicales en el estado de Veracruz. Tesis de Maestría. Veracruz, Ver., México. Universidad Veracruzana. Facultad de Medicina Veterinaria y Zootecnia. $88 \mathrm{p}$.

DiTomaso, JM. 2000. Invasive weeds in rangelands: species, impacts, and management. Weed Science 48:255-265.

DiTomaso, JM; Drewitz, JJ; Kyser, GB. 2008. Jubatagrass (Cortaderia jubata) control using chemical and mechanical methods. Invasive Plant Science and Management 1:82-90.

Enloe, SF; Kyser, GB; Dewey, SA; Peterson, V; DiTomaso, JM. 2008. Russian knapweed (Acroptilon repens) control with low rates of aminopyralid on range and pasture. Invasive Plant Science and Management 1:385-389.

Enríquez Q, JF; Meléndez N, F; Bolaños A, ED. 1999. Tecnología para la producción y manejo de forrajes tropicales en México. Libro Técnico Núm. 7. División Pecuaria. INIFAP. CIRGOC. Campo Experimental Papaloapan, Veracruz, México. 262 p.

Esqueda, VA. 2001. Efecto de la mezcla formulada picloram + fluroxypir en hierba dulce [Lippia nodiflora (L.) Michx.] y puzgual (Croton cortesianus Kunth) en potreros. In: Guevara F, F. ed. Memoria XXII Congreso Nacional de la Ciencia de la Maleza. Colima, Col., México. p. 57-62.

Esqueda, VA. 2003. Evaluación de los herbicidas Crosser y Vaquero en el control de malezas herbáceas en pastizales tropicales. Agronomía Mesoamericana 14:177-183.

Esqueda, VA; Tosquy V, OH; Rosales R, E. 2005. Efectividad de la mezcla picloram y fluroxipir en el control de malezas perennes de pastizales tropicales. Agronomía Mesoamericana 16:187-192.

Esqueda, VA; Tosquy, OH. 2007. Efectividad de métodos de control de malezas en la producción de forraje del pasto Pangola (Digitaria decumbens Stent.). Agronomía Mesoamericana 18:1-10.

Esqueda, VA; Montero, M; Juárez, FI. 2009. Efecto de métodos de control de malezas en la productividad y calidad del pasto Estrella de África (Cynodon plectostachyus (K. Schum.) Pilg. Tropical and Subtropical Agroecosystems 10(3):393-404.

Frans, RR; Talbert, R; Marx, D; Crowley, H. 1986. Experimental design and techniques for measuring and analyzing plant responses to weed control practices. In: Camper, ND ed. Research methods in weed science. 3 ed. Southern Weed Science Society. Champaign IL, USA. p. 29-46. 
García, E. 1987. Modificaciones al sistema de clasificación climática de Köppen. 4 ed. Universidad Nacional Autónoma de México. México, D. F. 130 p.

Goering, HK; Van Soest, PJ. 1970. Forage fiber analysis (apparatus, reagents, procedures, and some applications). Agric. Handbook No. 379. United States Department of Agriculture. Agricultural Research Service. Washington, DC, USA. 20 p.

Gomez, KA; Gomez, AA. 1984. Statistical procedures for agricultural research. 2 ed. J. Wiley \& Sons. New York, USA. 680 p.

Grice, AC; Campbell, SD. 2000. Weeds in pasture ecosystems - symptom or disease? Tropical Grasslands 34:264-270

Guevara, S; Meave, J; Moreno-Casasola, P; Laborde, J; Castillo, S. 1994. Vegetación y flora de potreros en la sierra de Los Tuxtlas. Acta Botánica Mexicana 28:1-27.

Harker, KN; Baron, VS; Chanasyk, DS; Naeth, MA; Stevenson, FC. 2000. Grazing intensity effects on weed populations in annual and perennial pasture systems. Weed Science 48:231-238.

Juarez-Lagunes, FI; Fox, DG; Blake, RW; Pell, AN. 1999. Evaluation of tropical grasses for milk production by dual-purpose cows in tropical Mexico. Journal of Dairy Science 82:2136-2145.

Juárez, FI; Montero LM; Serna, C; Alpírez, F; Canudas, EG. 2005. Evaluación de gramíneas forrajeras tropicales para bovinos en el centro del estado de Veracruz. In: Barradas L, HV. ed. Avances en la investigación agrícola, pecuaria, forestal y acuícola en el trópico mexicano. Libro Científico No. 2. INIFAP. UV. CP. UACH. ITUG. ITBOCA. UNAM. Veracruz, México. p. 195-204.

Koger, CH; Stritzke, JF; Cummings, DC. 2006. Control of sericea lespedeza (Lespedeza cuneata) with triclopyr, fluroxypyr, and metsulfuron. Weed Technology 16:893-900.

Mertens, DR. 1994. Regulation of forage intake. In: Fahey Jr, GC; Collins, M; Mertens D R; Moser LE. eds. Forage quality, evaluation and utilization. American Society of Agronomy. Crop Science Society of America. Soil Science Society of America. Madison, WI, USA. p. 450-493

Middleton, CH. 1982. Dry matter and nitrogen changes in five tropical grasses as influenced by cutting height and frequency. Tropical Grasslands 16:112-117.

Miller, IL. 1992. Competition between Brachiaria humidicola and Mimosa pigra. Tropical Grasslands 26:111-114.

Motooka, PS. 1986. Chemical weed control in tropical pastures. In: Moody, K. ed. Weed control in tropical crops. Vol. II. Weed Science Society of the Philippines. Southeast Asian Regional Center for Graduate Study and Research in Agriculture. Los Baños, Laguna, Philippines. p. 9-54.

Norman, EM; Gunn, CR. 1985. Pachecoa prismatica (Fabaceae): taxonomy and phylogeny. Brittonia 37:78-84.

Panter, KE; James, IF. 1990. Natural plant toxicants in milk: a review. Journal of Animal Science 68:892-904.

Pellegrini, LG; Nabinger, C; Faccio C, PC; Neumann, M. 2007. Diferentes métodos de controle de plantas indesejáveis em pastagem nativa. Revista Brasileira de Zootecnia 36:1247-1254.

Penati, MA; Corsi, M; Gonçalves, LC; Bueno, MG Jr; Silva, DCT. 2005. Número de amostras e relação dimensão: formato da moldura de amostragem para determinação da massa de forragem de gramíneas cespitosas. Revista Brasileira de Zootecnia 34:36-43.

Peña, ME; Urdaneta, F; Arteaga, G; Casanova, A. 1999. Relación del manejo de pastizales con las zonas agroecológicas en los municipios Rosario y Machiques de Perijá, estado Zulia, Venezuela. Revista de la Facultad de Agronomía (LUZ) 16(Supl. 1):213-219.

Pinzón, B; Montenegro, R. 1988. Efecto de los herbicidas en el combate de malezas de hoja ancha en potreros del área de Gualaca, Provincia de Chiriquí, República de Panamá. Ciencia Agropecuaria 5:61-76.

Rosario L, MI. 2005. Efectos de la calidad del forraje y el tipo de concentrado sobre el consumo voluntario y producción de leche en vacas lecheras. Tesis de Maestría. Mayagüez, Puerto Rico. Universidad de Puerto Rico. Recinto Universitario de Mayagüez. 64 p.

Samuel, LW; Lym, RG. 2008. Aminopyralid effects on Canada thistle (Cirsium arvense) and native plant species. Invasive Plant Science and Management 1:265-278.

Sanders, GE; Pallett, KE. 1987. Comparison of the uptake, movement, and metabolism of fluroxypyr in Stellaria media and Viola arvensis. Weed Research 27:159-166.

Scanlan, JC. 1984. Herbicidal control of woody weeds in central Queensland. 1. Brigalow (Acacia harpophylla). Tropical Grasslands18:26-32.

Serna G, C. 2004. Evaluación nutricional de gramíneas forrajeras tropicales para bovinos en el centro del estado de Veracruz. Tesis de Maestría. Veracruz, Veracruz, México. Universidad Veracruzana. Facultad de Medicina Veterinaria y Zootecnia. $151 \mathrm{p}$.

Sheley, RL; Jacobs, JS; Carpinelli, MF. 1998. Distribution, biology, and management of diffuse knapweed (Centaurea diffusa) and spotted knapweed (Centaurea maculosa). Weed Science 12:353-362. 
Sosa, L; Medrano, C. 1992. Evaluación de herbicidas para el control de malezas arbustivas en pastizales del estado Zulia. Revista de la Facultad de Agronomía (LUZ) 9: 199-212.

Thomson, WT. 1993. Agricultural chemicals. Book II Herbicides. Thomson Publications. Fresno, CA, USA. 310 p.

Timmons, FL. 1970. A history of weed control in the United States and Canada. Weed Science 18:294-307.

Valbuena, NJ; Acosta, C. 2006. Control de malezas dicotiledóneas en los rendimientos del pasto Estrella (Cynodon nlemfuensis Vanderyst). In: Tejos M, R. ed.
Memoria X Seminario Manejo y utilización de pastos y forrajes en sistemas de producción animal. Maracaibo, Venezuela. p. 34-42.

Van Soest, PJ. 1994. Nutritional ecology of the ruminant. 2 ed. Cornell University Press. Ithaca, NY, USA. 476 p.

Villaseñor, JL; Espinosa G, FJ. 1998. Catálogo de malezas de México. Universidad Nacional Autónoma de México. Consejo Nacional Consultivo Fitosanitario. Fondo de Cultura Económica. México, D. F. 449 p.

Vitelli, JS. 2000. Options for effective weed management. Tropical Grasslands 34:280-294. 